\title{
Persistent Infrared Hole Burning of Ammonium Stearate
}

\author{
Gu-Sheng Yu, ${ }^{\dagger}$ Hung-Wen Li, and Herbert L. Strauss* \\ Department of Chemistry, University of California, Berkeley, California 94720-1460
}

Received: June 20, $1997^{\otimes}$

\begin{abstract}
Preliminary analysis of the X-ray diffraction of single crystals provides the unit cell parameters and suggests standard B-type packing of the polymethylene chains. Spectral hole burning of the N-D stretching bands of ammonium stearate, doped with some deuterium, produces holes and antiholes in three regions of the spectrum. The three regions contain the stretching bands, the $\mathrm{NH}_{3}$ rocking bands, and the rocking-libration bands, respectively. The holes and antiholes identify the vibrational bands and correlate them with the different possible orientations of the $\mathrm{NH}_{3} \mathrm{D}^{+}$in the crystal.
\end{abstract}

\section{Introduction}

The salts of fatty acids are one in a series of compounds that serve as models for various lipids. ${ }^{1}$ They are also commonplace and familiar as soaps. The details of the crystal structure of these compounds are important as an indicator of more complicated molecules, and the vibrational spectra are useful for understanding the spectra of the entire range of methylene chain compounds.

We have developed the infrared hole-burning technique to identify bands in the infrared spectrum and often to assign them to various conformers or crystal structures. ${ }^{2}$ We have been able to use this technique on a large number of different ammonium salts doped with a small amount of deuterium. This typically gives rise to a spectrum with one $\mathrm{N}-\mathrm{D}$ band for each distinct orientation of the $\mathrm{NH}_{3} \mathrm{D}^{+}$ion. There are usually four distinct orientations and four N-D bands. Previously, we have burned $\mathrm{N}-\mathrm{D}$ stretching bands and observed the changes in only the stretching region of the spectrum. These changes result from the reorientation of the ions and are evidenced by the decrease in the $\mathrm{N}-\mathrm{D}$ band belonging to irradiated orientation and increases in the other three bands. Here we investigate ammonium stearate. We burn the stretching bands and observe changes in the stretching region of the spectrum. Since the process of hole burning involves reorientation of the ions, all the bands arising from the $\mathrm{NH}_{3} \mathrm{D}^{+}$should change. We expect changes in the spectral regions containing the ammonium ion deformation, and indeed, we observe these as well.

Many of the physical properties of this salt are determined by the long aliphatic chain. ${ }^{1}$ It has been generally accepted that the potassium soaps crystallize in the triclinic B-form for $n$, the number of carbon atoms in the chain, an even number from 12 to $18 .^{1,3,4}$ The B-form transforms to the monoclinic C-form at about $65{ }^{\circ} \mathrm{C}$. ${ }^{1,4}$ (The exact temperature reported depends on the method used for the determination..$^{5}$ ) The ammonium ion occupies about the same volume as does the potassium, and so it is reasonable to expect a similar structure. Of course, the ammonium ion has the capability of hydrogen bonding, and it is interesting to see what difference this makes.

In this paper we start with a preliminary analysis of the crystal structure. We then present the results of the hole burning of the $\mathrm{N}-\mathrm{D}$ bands of the $\mathrm{NH}_{3} \mathrm{D}^{+}$, dilute in the ammonium stearate, and show how the burning of the N-D bands allows the assignment of $\mathrm{N}-\mathrm{D}$ bands in a number of regions of the spectrum.

$\dagger$ Present address: Industrial Technology Research Institute-Center for Measurement Standards, 321 Kuang Fu Rd, Section 2, Hsinchu, Taiwan 30042, ROC.

${ }^{\otimes}$ Abstract published in Advance ACS Abstracts, October 1, 1997.

\section{Experimental Section}

We have grown crystals of the ammonium salts of a number of fatty acids. However, we found we could not analyze the diffraction pattern of the stearate past deriving the unit cell. We can compare the unit cell for the stearate with that of the palmitate and the myristate and guess at the approximate structure. The details of the crystal structures of these compounds and the relationship of the structure to the infrared spectra will be considered in a later paper. ${ }^{6}$

The unit cell for the stearate is triclinic with $a=4.174 \AA, b$ $=73.93 \AA$, and $c=6.363 \AA$ with $\alpha=86.083^{\circ}, \beta=101.943^{\circ}$, and $\gamma=92.380^{\circ}$ (at $150 \mathrm{~K}$ ). These dimensions are very similar to those of the palmitate and myristate except that $b_{\text {stearate }}>$ $b_{\text {palmitate }}>b_{\text {myristate }}$ to accommodate the longer chains of the larger molecules. The areas of the $a-c$ plane of the unit cell for these three molecules are almost identical and for the stearate, palmitate, and myristate are 25.98, 25.99, and 26.02 $\AA^{2}$, respectively. Similarly, the $\beta$ angles are almost identical, $101.94^{\circ}, 102.02^{\circ}$, and $101.92^{\circ}$, respectively. The methylene chains in the palmitate and the myristate are, as expected, in the B-form.

For our present purposes, we will assume that the methylene chains of the stearate are also arranged in a triclinic form similar to the B-form of the potassium salts and that the ammonium ions occupy positions similar to the potassium ions. We note that, in order to hole burn using the ammonium ion bands, the ions must be locked in a definite position and not rotate rapidly on the time scale of our experiments. We see no evidence for a phase transition as we lower the temperature to $7 \mathrm{~K}$, and we will assume that the crystal remains in the B-form.

The preparation of fatty acid salt crystals is subject to contamination by the half-acids. ${ }^{7}$ The salt crystals are prepared by adding excess ammonium hydroxide to warm alcoholic solutions of the acid. A small amount of $\mathrm{D}_{2} \mathrm{O}$ had been added to the ammonium hydroxide. The resulting solution is then placed in a sealed vial and allowed to cool to form crystals. These crystals are dried in a vacuum, in either a desiccator or a closed-cycle refrigerator. To put the crystals in the refrigerator, the crystals are spread evenly between two $\mathrm{KBr}$ windows, and the resulting sandwich was mounted. The room-temperature spectrum is checked to ensure that the solvent has been removed and that no half-acid is present. The temperature is then lowered to $7 \mathrm{~K}$ over the course of a few hours and the sample left to equilibrate for many hours before the hole burning is attempted.

The apparatus used is similar to that described in our previous papers ${ }^{8}$ and now consists of a Nicolet IR-850 FTIR spectrometer fitted with both MCT/B and InSb detectors. The spectrometer 
was run at $1 \mathrm{~cm}^{-1}$ resolution. (The resolution stated is that before apodization.) The laser system used to produce the infrared radiation for the hole burning has been described previously and has a spectral bandwidth of about $1 \mathrm{~cm}^{-1} .9$ The $\mathrm{N}-\mathrm{D}$ stretching bands of the ammonium stearate burn rather easily, and we can use 5 min irradiation time for many of the spectra. For the higher frequency spectra (wavenumbers above about $2000 \mathrm{~cm}^{-1}$ ), we use the InSb detector and average 64 scans. For lower frequency spectra, we irradiate the same N-D stretching bands for $30 \mathrm{~min}$ to obtain deeper holes and then examine the results with the MCT detector using 4096 scans for each spectrum. This many scans takes $100 \mathrm{~min}$. The decay of the holes at $7 \mathrm{~K}$ is slow enough to allow these long scan times. After each hole-burning experiment, the temperature is raised to $90 \mathrm{~K}-\mathrm{a}$ process that takes about a half-hour. The sample is then cooled back to $7 \mathrm{~K}$ and left to equilibrate before starting again.

All the spectra are taken "single beam", and so a reference spectrum must be subtracted from each observed spectrum to obtain the absorption spectra displayed below. For many situations, the reference spectrum is obvious (and is indicated in the figure captions), and if the spectrometer changes in any way, the reference spectrum can often be multiplied by a factor close to 1 to compensate. Where this is not possible-for example, when we need to examine both large and small bands arising from different isotomers - the uncertainty in the proper subtraction increases the errors in determining the size of the band or hole.

\section{Results}

ND Stretching Region. The putative N-D band region near $2200 \mathrm{~cm}^{-1}$ was irradiated near the peaks in the absorption spectrum, with the results shown in Figure 1. The bottom panel shows the difference between a deuterated and a nondeuterated sample of ammonium stearate. The panel shows the fit of the observed spectrum to the sum of four Lorenztian bands. The fit was simply to the absorption spectrum in an attempt to find the expected four $\mathrm{N}-\mathrm{D}$ stretching bands. The top panels show the results of the hole burning at the irradiation wavenumbers, $\omega_{\mathrm{B}}$, listed. Each spectrum is the difference between the spectrum after irradiation and that before. Note that the holes and antiholes are not necessarily at the wavenumbers of the irradiation. The irradiation wavenumbers are 2213, 2222, 2226, and $2234 \mathrm{~cm}^{-1}$, while the holes are at 2217, 2219, 2227, and $2234 \mathrm{~cm}^{-1}$. The shift for the first two bands comes from severe overlap of the absorption bands. The hole burning provides resolution of the overlapped bands.

The relative intensity of the four absorption bands arises from two effects: the energy of the $\mathrm{NH}_{3} \mathrm{D}^{+}$ion is slightly different in each of the four orientations, resulting in different Boltzmann factors at low temperatures, and the intrinsic intensity of a hydrogen-bonded band varies with the strength of the bond and its vibrational frequency. The relative intensities are $12 \%$ for the $2217 \mathrm{~cm}^{-1}$ band, $20 \%$ for $2219 \mathrm{~cm}^{-1}, 43 \%$ for $2227 \mathrm{~cm}^{-1}$, and $25 \%$ for $2234 \mathrm{~cm}^{-1}$. The widths vary systematically, as in many hydrogen-bonded systems, ${ }^{2}$ and the full width at halfmaximum (fwhm) is $8.9 \mathrm{~cm}^{-1}$ for the $2217 \mathrm{~cm}^{-1}$ band, $7.6 \mathrm{~cm}^{-1}$ for $2219 \mathrm{~cm}^{-1}, 7.2 \mathrm{~cm}^{-1}$ for $2227 \mathrm{~cm}^{-1}$, and $7.1 \mathrm{~cm}^{-1}$ for 2234 $\mathrm{cm}^{-1}$.

When the laser is set to the frequency of one of the absorption bands indicated by the curve fitting, the resulting hole is at the frequency of the laser. This shows that the position of the bands indicated by the curve fitting is indeed the position of the various $\mathrm{N}-\mathrm{D}$ stretches. After $5 \mathrm{~min}$ of irradiation, the hole tends to broaden and merge with the neighboring hole. Figure 1 shows the results of purposely moving the laser wavenumbers off the

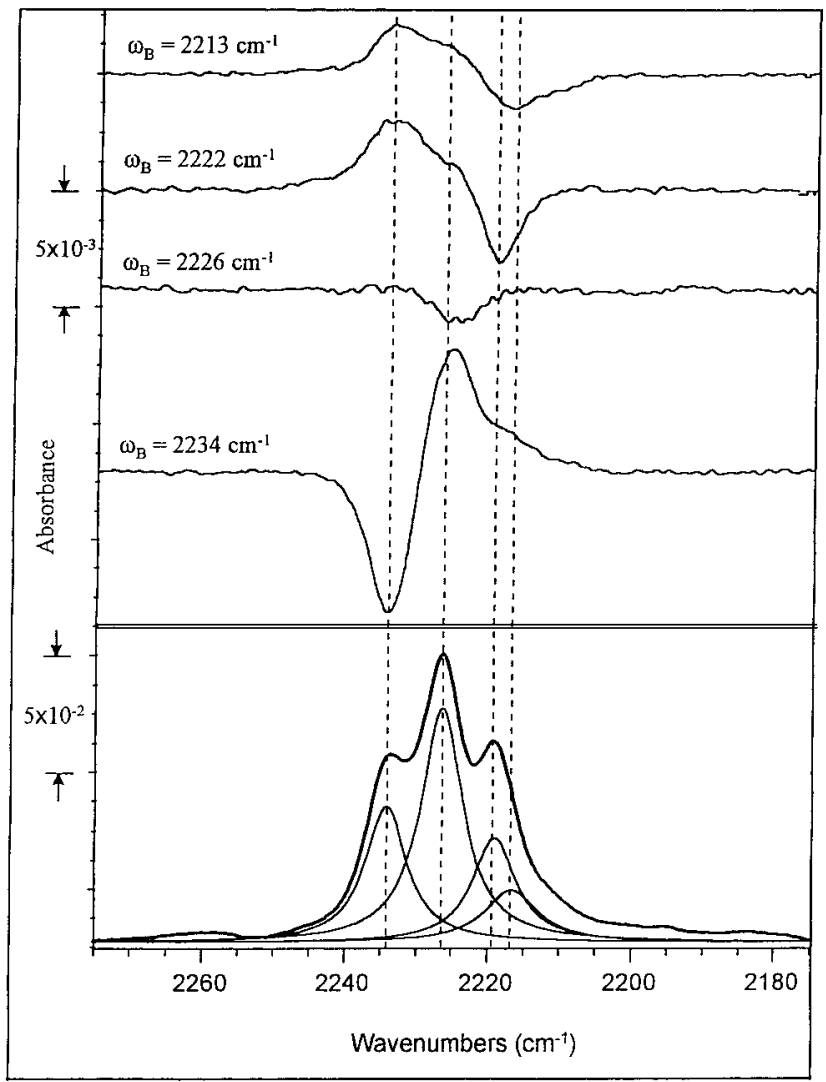

Figure 1. Infrared spectra of ammonium stearate containing ammonium groups doped with $4 \%$ deuterium in the $\mathrm{N}-\mathrm{D}$ stretching region. The bottom panel shows the difference between spectra of the deuterated and the nondeuterated compound. It also shows the four component bands obtained by curve fitting the observed difference spectrum. The top panel shows the hole-burning spectra obtained as the difference between the spectrum after and before laser irradiation at the wavenumbers $\omega_{\mathrm{B}}$. The laser positions at 2213 and $2222 \mathrm{~cm}^{-1}$ were purposely offset from the center of the corresponding holes to show that such centers could be determined. The laser irradiation time was $5 \mathrm{~min}$. The fiducial lines are at the centers of the bands identified by curve fitting and are at 2217, 2219, 2226, and $2233 \mathrm{~cm}^{-1}$.

center of the bands (for 2213 and $2222 \mathrm{~cm}^{-1}$ ) to show that the initially formed holes indicate the true position of the bands. The efficiency of the hole burning varies from band to band, with the band at $2234 \mathrm{~cm}^{-1}$ giving the deepest hole and that at $2227 \mathrm{~cm}^{-1}$ the shallowest, for constant irradiation time.

Mid-Infrared. To see the spectrum below $2000 \mathrm{~cm}^{-1}$, we switch to the MCT/B detector. The spectrum of deuterated ammonium stearate in the mid-infrared is shown in Figure 2 (bottom panel).

Previous studies have identified the normal coordinates of the fatty acids - alkaline metal salts with the various regions of the spectrum. We follow this classification using assignments of the potassium salts. ${ }^{10}$ The strong wide band between 1600 and $1500 \mathrm{~cm}^{-1}$ contains the $\mathrm{COO}$ asymmetric stretch and in the region between 1500 and $1400 \mathrm{~cm}^{-1}$ is the corresponding symmetric stretch and some of the $\mathrm{CH}_{2}$ scissors motions. The $\mathrm{CH}_{2}$ wags appear in a characteristic progression below 1400 $\mathrm{cm}^{-1}$.

In addition, there are bands associated with $\mathrm{NH}_{4}{ }^{+}$and with the small amount of $\mathrm{NH}_{3} \mathrm{D}^{+}$. It is easiest to guess where these might be by comparing with the well-known frequencies of the isotopically substituted methanes. ${ }^{11}$ Relevant methane vibrations are for $\mathrm{CH}_{4}$ the degenerate deformation at $1534 \mathrm{~cm}^{-1}$ and for $\mathrm{CH}_{3} \mathrm{D}$ the $\mathrm{CD}$ stretch at $2200 \mathrm{~cm}^{-1}$, the symmetric deformation at $1300 \mathrm{~cm}^{-1}$, and the degenerate (asymmetric) $\mathrm{CH}_{3}$ deformation or rocking bend at $1155 \mathrm{~cm}^{-1}$. The various $\mathrm{N}-\mathrm{D}$ stretches are at wavenumbers just a bit above the position of the $\mathrm{C}-\mathrm{D}$ stretch 


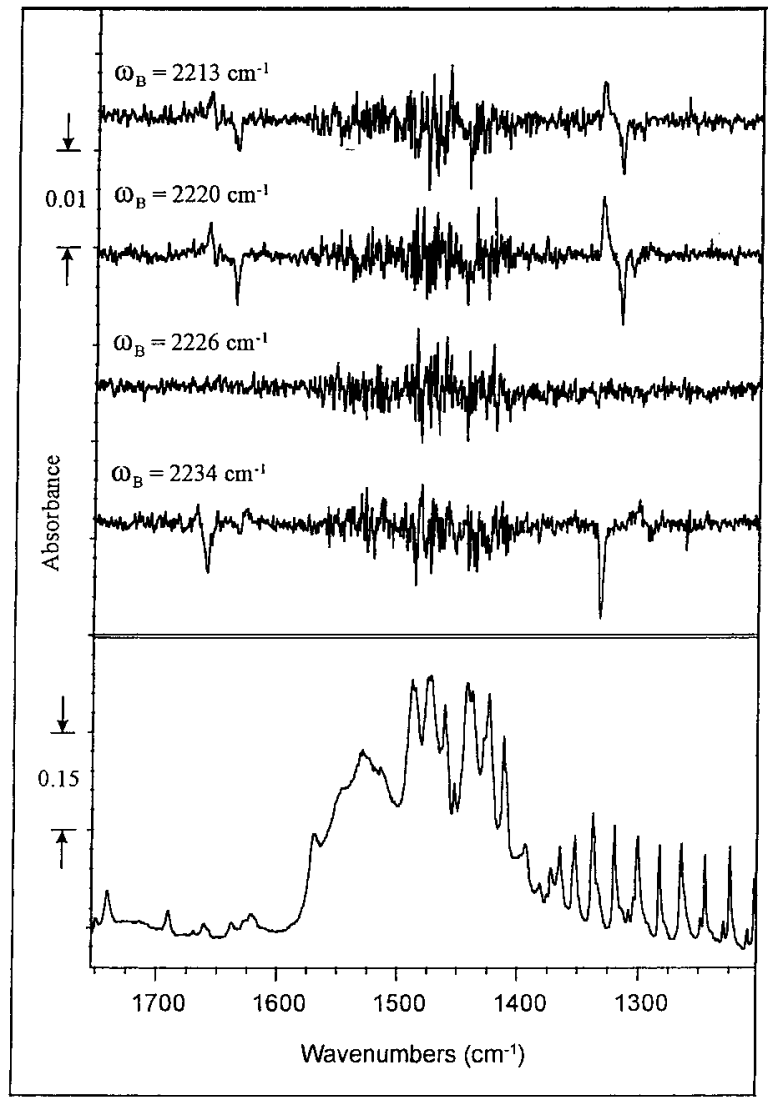

Figure 2. Infrared spectra in the mid-infrared. The bottom panel shows the spectrum of ammonium stearate containing a small amount of deuterium. The top panel shows the results of hole burning at the center of the $\mathrm{N}-\mathrm{D}$ stretching bands identified in Figure 1. The laser irradiation time was $30 \mathrm{~min}$.

(Figure 1). For comparison, N-D bands less strongly hydrogen bound would appear near $2400 \mathrm{~cm}^{-1}$. $^{12,13}$ For the various deformations, no direct comparison of methane with $\mathrm{NH}_{4}{ }^{+}$has yet been made. The degenerate $\mathrm{NH}_{3}$ deformation (also called the ND bend) has been seen assigned at about $1260 \mathrm{~cm}^{-1} \cdot{ }^{14}$ Again, a change in hydrogen bonding will change this frequency.

The hole-burning process rotates the ammonium ion from one orientation to another, producing holes and antiholes. The various lower frequency vibrations should follow; that is, the deformation band of orientation 1 should yield a hole on burning the stretch band 1 . The pattern of holes and antiholes immediately assigns the bands of the deformations to the $\mathrm{NH}_{3} \mathrm{D}^{+}$ ions in definite orientations. The results of hole burning on the various lower frequency parts of the spectrum are shown as difference plots in the upper part of Figure 2. Holes and antiholes show up in two regions, 1310-1340 and 1640-1660 $\mathrm{cm}^{-1}$. These two regions are shown on an expanded scale in Figures 3 and 4.

The bands near $1330 \mathrm{~cm}^{-1}$ are the $\mathrm{NH}_{3}$ deformations. In Figures 3 and 4, the dashed fiducial lines drawn to allow comparison of the various features of the absorption and hole spectra are not necessarily through the center of either the apparent holes or the absorption features. The apparent shape of the holes is influenced by the considerable amount of noise. In Figure 3, we see that the first reference line at $1308.2 \mathrm{~cm}^{-1}$ goes near the minimum in the hole produced by the $2220 \mathrm{~cm}^{-1}$ radiation and is close to the maximum in the absorption feature at $1308 \mathrm{~cm}^{-1}$. The next line at $1317.5 \mathrm{~cm}^{-1}$ goes through the minimum in the hole produced by the $2220 \mathrm{~cm}^{-1}$ radiation and is close to that produced at $\sim 1317.2 \mathrm{~cm}^{-1}$ by the $2213 \mathrm{~cm}^{-1}$ radiation. It shows that the shoulder at about this wavenumber in the absorption spectrum is the $\mathrm{NH}_{3} \mathrm{D}^{+}$deformation. The line

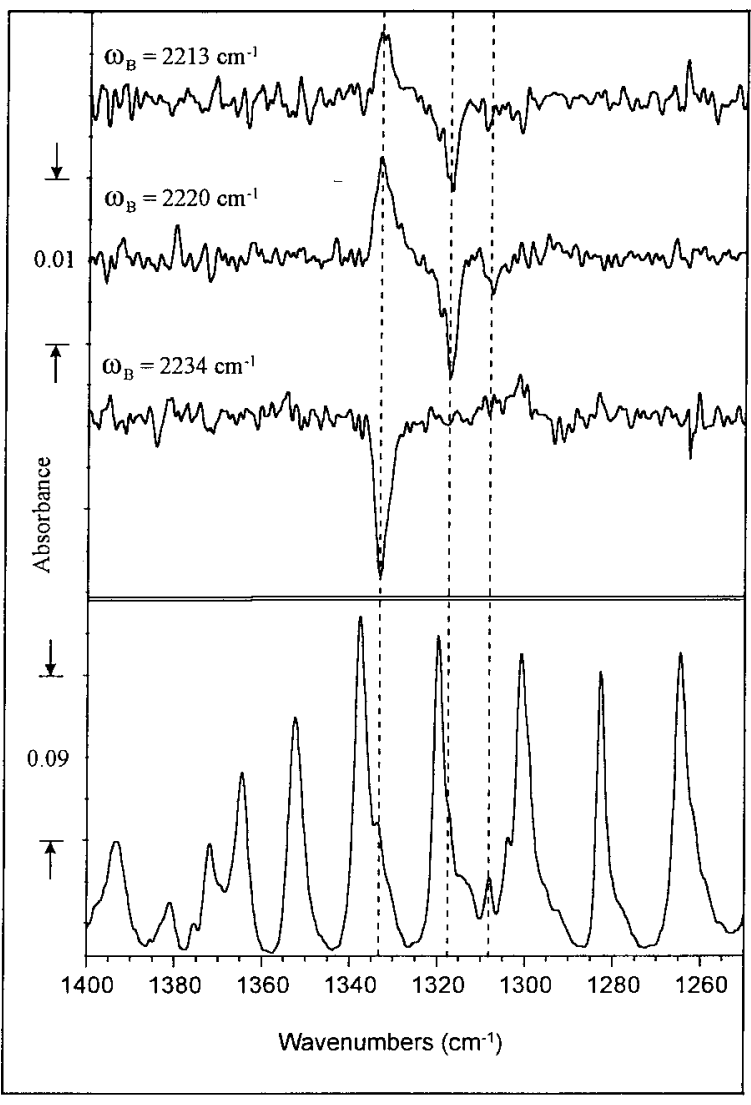

Figure 3. Part of Figure 2 on an expanded scale from 1250 to 1400 $\mathrm{cm}^{-1}$. This shows the $\mathrm{NH}_{3} \mathrm{D}^{+}$symmetric deformation bands. Note that the difference spectrum produced by irradiation at $2226 \mathrm{~cm}^{-1}$ shows no holes and is not shown. The fiducial lines are at 1308.2, 1317.5, and $1333.2 \mathrm{~cm}^{-1}$ and are close to the center of various hole and absorption features.

at $1333 \mathrm{~cm}^{-1}$ indicates another absorption shoulder as a deformation.

The combination bands and holes in Figure 4 are noisier. The first line at $1638.6 \mathrm{~cm}^{-1}$ goes close to the center of the holes and an absorption feature. For the other combinations expected, things are not so clear. The holes indicated near $1660.9 \mathrm{~cm}^{-1}$ do line up with an absorption shoulder. The line at $1669.6 \mathrm{~cm}^{-1}$ goes through an absorption feature and through a questionable antihole produced by the $2234 \mathrm{~cm}^{-1}$ radiation.

Comparing the two regions in Figure 2 or in Figure 3 and Figure 4 shows that the pattern of the hole burning repeats. We assign the bands near $1650 \mathrm{~cm}^{-1}$ to combinations of the corresponding bands near $1330 \mathrm{~cm}^{-1}$ with another mode (Table 1). Comparing frequencies suggests that this mode is the $\mathrm{NH}_{3} \mathrm{D}^{+}$libration with a frequency of about $325 \mathrm{~cm}^{-1}$. Note that the librational modes can be different for each orientation of the ion. The frequency matches the libration frequency of $325 \mathrm{~cm}^{-1}$ we found for the libration in the Tutton salts. ${ }^{15}$ Table 1 also matches the deformations in each orientation of the $\mathrm{NH}_{3} \mathrm{D}^{+}$with the corresponding $\mathrm{N}-\mathrm{D}$ stretch and thus with a given $\mathrm{NH}_{3} \mathrm{D}^{+}$orientation. We have assigned the bands at 1317.2, $1317.5 \mathrm{~cm}^{-1}$ and 1638.1, $1638.6 \mathrm{~cm}^{-1}$ to separate deformations and their corresponding combinations. This part of the assignment is uncertain. We expect four of each type of band but have not been able to find them-especially those that correlate with the $2226.5 \mathrm{~cm}^{-1}$ stretch. Finally, we note that the bending bands at about $1300 \mathrm{~cm}^{-1}$ should be doubly degenerate. The symmetry is low enough to lift the degeneracy, and so each band could be doubled. To find all the split bands will require much better signal-to-noise ratios.

The power of the hole-burning technique to assign small features of the spectrum is illustrated in the figures. In Figure 
TABLE 1: Persistent Infrared Hole-Burning Parameters of $\mathrm{NH}_{3} \mathrm{D}^{+}$in Ammonium Stearate

\begin{tabular}{|c|c|c|c|c|c|c|c|c|c|c|c|c|c|}
\hline \multirow[b]{2}{*}{$\mathrm{NH}_{3} \mathrm{D}^{+}$} & \multicolumn{3}{|c|}{$\begin{array}{c}\operatorname{stretch}\left(v_{1}\right) \\
\left(2 \mathrm{~min} \text { hole burn at } v_{1}\right)\end{array}$} & \multicolumn{3}{|c|}{$\begin{array}{c}\operatorname{stretch}\left(v_{1}\right) \\
\left(30 \mathrm{~min} \text { hole burn at } v_{1}\right)\end{array}$} & \multicolumn{3}{|c|}{$\begin{array}{c}\text { combination } \\
\left(30 \text { min hole burn at } v_{1}\right)\end{array}$} & \multicolumn{3}{|c|}{$\begin{array}{c}\text { deformation } \\
\left(30 \text { min hole burn at } v_{1}\right)\end{array}$} & \multirow{2}{*}{ 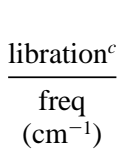 } \\
\hline & $\begin{array}{c}\text { freq } \\
\left(\mathrm{cm}^{-1}\right)\end{array}$ & $\begin{array}{l}\text { fwhm }^{a} \\
\left(\mathrm{~cm}^{-1}\right)\end{array}$ & $\begin{array}{c}\Delta A / A^{b} \\
\left(\times 10^{-2}\right)\end{array}$ & $\begin{array}{c}\text { freq } \\
\left(\mathrm{cm}^{-1}\right)\end{array}$ & $\begin{array}{l}\text { fwhm } \\
\left(\mathrm{cm}^{-1}\right)\end{array}$ & $\begin{array}{c}\Delta A / A \\
\left(\times 10^{-2}\right)\end{array}$ & $\begin{array}{c}\text { freq } \\
\left(\mathrm{cm}^{-1}\right)\end{array}$ & $\begin{array}{l}\text { fwhm } \\
\left(\mathrm{cm}^{-1}\right)\end{array}$ & $\begin{array}{c}\Delta A / A \\
\left(\times 10^{-2}\right)\end{array}$ & $\begin{array}{c}\text { freq } \\
\left(\mathrm{cm}^{-1}\right)\end{array}$ & $\begin{array}{l}\text { fwhm } \\
\left(\mathrm{cm}^{-1}\right)\end{array}$ & $\begin{array}{c}\Delta A / A \\
\left(\times 10^{-2}\right)\end{array}$ & \\
\hline 1 & 2233.3 & $7.5(3.4)^{d}$ & & 2234.8 & $7.9(3.5)$ & 23 & 1660.9 & $4.5(2.7)$ & 27 & 1333.2 & $3.2(2.4)$ & 17 & 327.7 \\
\hline 2 & 2226.5 & $6.3(2.8)$ & 2.4 & 2225.8 & $6.8(3.1)$ & 2 & & & & & & & \\
\hline 3 & 2219.1 & $6.2(2.8)$ & 5.2 & 2219.2 & $7.6(3.4)$ & 24 & $1638.6^{e}$ & $3.4(2.1)$ & 25 & $1317.5^{\mathrm{e}}$ & $3.1(2.4)$ & 16 & 321.1 \\
\hline 4 & 2217.2 & $8.9(4.0)$ & 4.0 & 2217.7 & $9.3(4.2)$ & 20 & $1638.1^{e}$ & $4.5(2.8)$ & 15 & $1317.2^{e}$ & $3.2(2.4)$ & 10 & 320.9 \\
\hline
\end{tabular}

${ }^{a}$ fwhm: the full width at the half-maximum. ${ }^{b} \Delta A / A$ : the ratio of hole depth to the absorption height. ${ }^{c}$ The differences between the deformation and the combination frequencies. ${ }^{d}$ The number in the parentheses is $10^{3}$ times the ratio of the width to the center frequency. ${ }^{e}$ The deformation bands and their associated combinations are not clearly separated. The frequencies listed are the peak frequencies of the holes.

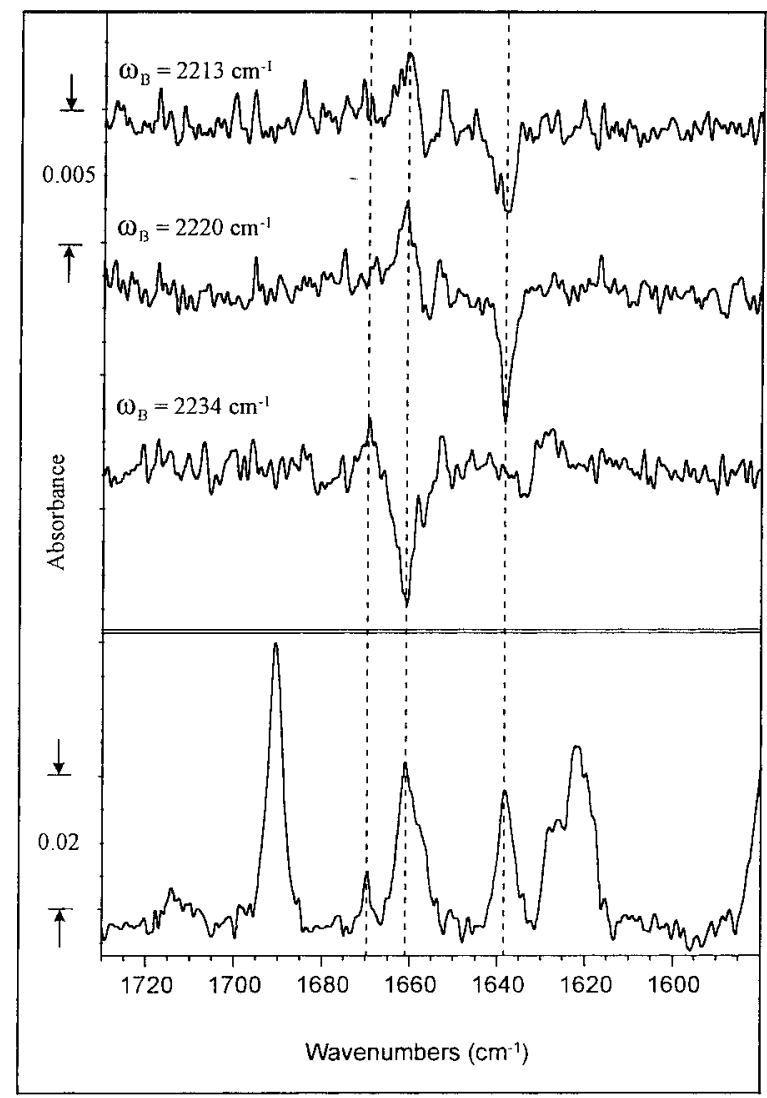

Figure 4. Part of Figure 2 as in Figure 3 but from 1580 to $1730 \mathrm{~cm}^{-1}$ to show the $\mathrm{NH}_{3} \mathrm{D}^{+}$combination bands. The fiducial lines are at 1638.6, 1660.9 , and $1669.6 \mathrm{~cm}^{-1}$.

3 the deformations of the small amount of $\mathrm{NH}_{3} \mathrm{D}^{+}$appear as small shoulders on much bigger bands in the absorption spectrum but are unambiguous from the hole-burning spectra. In Figure 4 the $\mathrm{NH}_{3} \mathrm{D}^{+}$absorption bands appear comparatively larger, but this is only because there are few fundamental bands of the methylene chain or of the $\mathrm{NH}_{4}{ }^{+}$in this region of the spectrum (see also Figure 2).

Hole Depths and Widths. If the holes at lower frequencies are indeed caused by driving the $\mathrm{NH}_{3} \mathrm{D}^{+}$ions to different orientations, the holes for the lower frequency vibrations should have the same fractional depth as those at the stretching frequencies. Table 1 shows that this condition is approximately satisfied. The appearance of the small deformation and combination bands is very sensitive to the choice of baseline, and the fractional intensities in the table are subject to appreciable errors. The band at $2226 \mathrm{~cm}^{-1}$ is considerably more difficult to burn, and irradiation at this frequency did not produce observable antiholes or observable changes in the deformation bands.

The holes are twice the homogeneous width of the lines in the limit of shallow hole burning. ${ }^{16}$ Our holes in the stretching region of the spectrum get broader with longer burn times (Table 1 ), as expected. We have no information on the lower frequency holes at short burn times, as we can only observe the holes at long burn times. The holes at the stretching frequencies and those for the deformations differ considerably in width. The holes at lower frequency are considerably narrower, and this shows that the homogeneous widths cannot be determined by the lifetime of the molecules in a given orientation. They can, of course, be due to any combination of $T_{1}$ or $T_{2}{ }^{\prime}$ (pure dephasing) times for the vibrations themselves. The widths are approximately a constant fraction of the frequency of the band, as Table 1 shows. The widths of the holes of the stretches do not agree with those obtained by curve fitting the absorption spectrum and are not a monotonically decreasing function of the frequency. This indicates that the holes we observe are the result of burning overlapping bands.

The hole widths are crudely a constant fraction of the frequencies of the various bands, and this seems to imply a simple mechanism for the width. We know that the widths are not purely homogeneous. (They are not in the short burn time limit.) We might try to correlate the widths (both homogeneous and inhomogeneous) to the hydrogen bond strengths. ${ }^{17,18}$ These widths, however, are likely due to both repulsive and attractive forces, the effect of which varies from mode to mode in the same molecule. ${ }^{19}$ We do not know of the simple mechanism that would produce the observed result.

Acknowledgment. We gratefully thank Dr. Fred Hollander for obtaining the X-ray parameters of the ammonium stearate and Dr. Robert G. Snyder for enlightening discussions. We are pleased to acknowledge support by the NSF (CHE 9526754).

\section{References and Notes}

(1) Small, D. M. The Physical Chemistry of Lipids; Plenum Press: New York, 1986; $672 \mathrm{pp}$.

(2) Strauss, H. L. Acc. Chem. Res 1997, 30, 37.

(3) Vand, V.; Lomer, T. R.; Lang, A. Acta Crystallogr. 1949, $2,214$.

(4) Lomer, T. R. Acta Crystallogr. 1952, 5, 11.

(5) Grant, R. F.; Durrell, B. A. Can. J. Chem. 1960, 38, 1951.

(6) Yu, G. S.; Li, H.-W.; Hollander, F.; Snyder, R. G.; Strauss, H. L., to be published.

(7) Kench, J. E.; Malkin, T. J. Chem. Soc. 1939, 230

(8) Fei, S.; Strauss, H. L. J. Phys. Chem. 1995, 99, 2256

(9) Kung, A. H.; Fei, S.; Strauss, H. L. Appl. Spectrosc. 1996, 50, 790.

(10) Garcia, M. V.; Redondo, M. I.; Cheda, J. A. R. Vib. Spectrosc. 1994, 6, 301.

(11) Shimanouchi, T. Tables of Molecular Vibrational Frequencies Consolidated; National Bureau of Standards: Washington, DC, 1972; Natl. Stand. Ref. Data Ser. Vol. I, p 39.

(12) Weier, J. E.; Strauss, H. L. J. Chem. Phys. 1993, 98, 4437.

(13) Roberts, M. P.; Strauss, H. L. J. Phys. Chem. 1987, 91, 4241.

(14) Oden, L. L.; Decius, J. J. Spectrosc. Acta 1964, $20,667$.

(15) Fei, S.; Yu, G. S.; Li, H. W.; Strauss, H. L. J. Chem. Phys. 1996, 104, 6394 .

(16) Voelker, S.; Macfarlane, R. M.; Genack, A. Z.; Trommsdorff, H. P.; van der Waals, J. H. J. Chem. Phys. 1977, 67, 1759.

(17) Cho, H. G.; Strauss, H. L. J. Chem. Phys. 1993, 99, 5661.

(18) Pimentel, C.; McClellan A. L. The Hydrogen Bond; Freeman: San Francisco, 1960; 475 pp.

(19) Schweitzer, K. S.; Chandler D. J. Chem. Phys. 1982, 76, 2296 